coloproctology $2021 \cdot 43: 223-228$

https://doi.org/10.1007/s00053-021-00537-1

Angenommen: 7. April 2021

Online publiziert: 30. April 2021

(c) Springer Medizin Verlag GmbH, ein Teil von Springer Nature 2021

\section{Hintergrund}

Jährlich erhalten mehr als 42.000 Menschen in der Bundesrepublik Deutschland eine Stomaanlage [1]. Spezialisierte Pflegefachkräfte unterstützen die anschließende Rehabilitation dieser Menschen mit dem Ziel der größtmöglichen Autonomie in der Stomaversorgung und der bestmöglichen Teilhabe am Lebensalltag. Seit 2006 erfolgt die umfangreiche und aufwändige Weiterbildung der Pflegekräfte zum Erwerb dieser Qualifikation nach einem einheitlichen Curriculum der Fachgesellschaft Stoma, Kontinenz und Wunde (FgSKW e.V.). Die Absolventinnen und Absolventen dieser Pflegefachweiterbildung führen die Berufsbezeichnung „Pflegeexperte Stoma, Kontinenz und Wunde" (PSKW). Informationen zum heutigen ganzheitlichen Aufgabenbereich, weit über die ursprünglich eigentliche Stomatherapie hinaus, finden sich im Berufsbild der PSKW ausführlich dargestellt [2]. Die Kernaufgaben der PSKW werden dort als Edukation der Stomaträgerinnen und Stomaträger mit den Methoden der Beratung, Schulung und Anleitung definiert. Diese Teilprozesse in der Unterstützung der Menschen mit einem Stoma werden im direkten und persönlichen Kontakt mit den Stomaträgerinnen und Stomaträgern in allen Phasen des Behandlungsverlaufs ausgeführt. Die fortschreitende Digitalisierung in den Arbeitsbereichen der PSKW ist seit Jahren spürbar. Der genaue Blick auf die Arbeitsprozesse dieser Pflegeexperten in den unterschiedlichen Sektoren des Ge-

Werner Droste · Margarete Wieczorek

FgSKW e.V., Selm, Deutschland

\title{
Digitalisierungsvorschub für spezialisierte Pflege?
}

\author{
Arbeitsbedingungen der Pflegeexperten \\ Stoma, Kontinenz und Wunde während der \\ SARS-CoV-2-Pandemie
}

sundheitswesens zeigt bereits heute viele Beispiele für den sinnvollen Einsatz digitaler Unterstützung. In den Akutkliniken existieren heute Dokumentationshilfen in digitaler Form. Auch in den Rehabilitationskliniken vollzog sich gleichermaßen die Umstellung auf eine digitale Dokumentation. Einen wahren Innovationsvorsprung erlebten die Pflegeexperten SKW jedoch eher im außerklinischen Bereich. Ausgelöst durch den zunehmenden Kostendruck im HomecareBereich, den steigenden gesetzlichen Anforderungen zur elektronischen Abrechnung mit den Kostenträgern und dem Wettbewerbsdruck mit der Notwendigkeit zur Effizienzsteigerung im Warenverkehr waren hier schon viel früher als im Klinikbereich enorme Investitionen notwendig. So bedeutet diese Entwicklung im Arbeitsalltag der meisten Pflegeexperten SKW im Homecare, dass die fachliche Dokumentation der Versorgungsprozesse genauso bereits vor Ort bei den Menschen zu Hause erledigt wird, wie auch die Organisation der notwendigen Belieferung mit medizinischen Hilfsmitteln. Selbstverständlich gehört die Fotodokumentation der Stomaanlage ebenfalls zum Dokumentationsumfang vor Ort.

Schon lange wurden in Modellvorhaben Wege der digitalisierten Kommunikation in der medizinischen Therapie und Pflege erprobt. Beispielsweise sei hier das Modell AGnES genannt. Im AGnESKonzept (arztentlastende, gemeindenahe, e-Health-gestützte, systemische Intervention) können Hausärzte Kranken- besuche und medizinische Tätigkeiten an qualifizierte Mitarbeiter delegieren und damit einen größeren Patientenstamm versorgen. Seit dem Jahr 2005 werden solche AGnES-Modellprojekte in verschiedenen Bundesländern mit unterschiedlichen Schwerpunkten erprobt [3]. Der Begriff e-Health („electronic health“) fasst dabei die Nutzung von gesundheitsbezogenen Technologien zusammen [4].

\section{Begriffliche Einordnung}

Zum besseren Verständnis gilt es, die jeweiligen Bezeichnungen zu verschiedenen telemedizinischen Anwendungen zu unterscheiden.

\section{Telemedizin}

Der Begriff Telemedizin definiert verschiedene Gesundheitsleistungen im Rahmen von Diagnostik, Therapie wie Prävention und Rehabilitation als auch in beratender Funktion in der medizinischen Versorgung von beteiligten Personen unter der Überbrückung von raum- und zeitunabhängiger Distanz [5].

\section{e-Health}

Der Begriff $e$-Health („electronic health“) wird mit sämtlicher Verbesserung in Qualität und Leistung als allgemeine Definition der Telemedizin gegenübergestellt [6]. Das Einsatzgebiet von e-Health konzentriert sich auf die Kommuni- 
kation, Information, Vernetzung sowie Dokumentation [7].

\section{Telepflege}

Die Telepflege (Telecare oder Telenursing) überträgt die Prinzipien der Telemedizin auf die Pflege. Pflegepersonen erbringen unter Anwendung von Informations- und Kommunikationstechnologien (IKT) pflegefachliche Gesundheitsleistungen. Dabei lassen sich präventive Maßnahmen, Informationen zum Pflegeprozess und zur pflegefachlichen Versorgung wie auch über Ereignisse in der poststationären Nachsorge kommunizieren. Das Instrument der Telepflege ermöglicht, die Nachsorge von Patientinnen und Patienten gezielt zu Hause zu unterstützen. Zudem sollen Gesundheitsleistungen in Form von Daten, Stimmen und Videos effizienter und ökonomischer gestaltet werden [8].

\section{Telefonberatung}

Die Telefonberatung stellt für Patientinnen und Patienten, die nicht die Möglichkeit einer persönlichen Beratung haben, eine Option dar [9]. Die Nursing Interventions Classification (NIC) definiert in der Klassifizierung von Therapien, die Krankenschwestern durchführen, die Telefonberatung als ,eliciting patient's concerns, listening, and providing support, information, or teaching in response to patient's stated concerns, over the telephone" [10].

\section{Studienlage zu Telecare}

Für diesen Beitrag wurden Studien untersucht, die eine Wirksamkeit der Teleberatung betrachten und/oder den Einsatz eines Videokonferenzsystems analysieren.

1. Auf dem Fachgebiet der Kardiologie betrachten Riegel et al. in einer randomisiert-kontrollierten klinischen Studie mit 358 Teilnehmern die Effektivität der telefonischen Fallberatung durch Krankenschwestern nach der Krankenhausentlassung von Patientinnen und Patienten mit chronischer Herzinsuffizienz. Die Ergebnisse weisen 6 Monate nach der Entlassung eine signifikant niedrigere Krankenhausaufenthaltsrate um 47,8\% sowie eine Reduzierung der stationären Kosten um 45,5\% auf. Folgend konnte mit Hilfe der Telefonberatung eine Kostenersparnis auf dem Gebiet erzielt und dem Drehtüreffekt vorgebeugt werden [11].

2. In einer weiteren Studie wurden in der Telepsychiatrie Videokonferenzsysteme zur Beratung von Patientinnen und Patienten in einer ländlichen Region in Kanada ausgewertet $(N=241)$. Zum Vergleich wurden dabei Patientinnen und Patienten in einem persönlichen Gespräch beraten $(N=254)$. Die Studie konzentriert sich auf die Gleichwertigkeit der beiden Formen der Leistungserbringung. Die psychiatrische Beratung und Telepsychiatrie führen in der Studie zu gleichwertigen Ergebnissen. Die beiden Gruppen zeigten sich zufrieden mit den Dienstleistungen. Hinsichtlich der Kosteneffektivität erwies sich die Telepsychiatrie sogar um $10 \%$ günstiger als die persönliche Beratung [12].

3. Inwiefern sich telefonische Kontakte kombiniert mit Teleberatung durch eine Fachkrankenschwester auf Veränderungen bei Patientinnen und Patienten auswirken, untersucht Hartford in einer randomisiertkontrollierten Telefoninterventionsstudie. In Bezug auf die körperlichen sowie psychischen Beschwerden als auch auf die Lebensweise zeigt sich eine beachtenswerte positive Auswirkung bei Patientinnen und Patienten nach einer Bypassoperation mit einer kurzen Verweildauer im Krankenhaus [13].

4. Um einen Unterschied zwischen telefonischer und keiner Intervention zu überprüfen, führten Piette et al. eine randomisiert-kontrollierten Studie mit 248 Diabetikerinnen und Diabetikern durch. In einem zweiwöchigen Intervall erfolgt eine telefonische Nachsorge durch die Diabetesfachkrankenschwester, bei der die Informationen nach der Gesundheit und Selbstversorgung eingeholt werden. Im Vergleich zu Diabetikerinnen und Diabetikern ohne Intervention steigt die Zufriedenheit sowie eine Verbesserung in der Gesundheitsversorgung bei den Teilnehmenden, die in kontinuierlichem telefonischem Kontakt mit der Diabetes-Fachkrankenschwester standen [14].

5. Tremont et al. präsentieren in ihrer randomisiert-kontrollierten Studie die Ergebnisse der pflegerischen Telefonberatung bei 250 pflegenden Angehörigen von Pflegebedürftigen mit einer diagnostizierten Demenz. Sie untersuchten die Auswirkung der telefonischen Intervention auf die Pflegenden. Über einen Zeitraum von 12 Monaten führten sie eine Teleberatung mit einzelnen Personen durch. Zu Beginn fand die Häufigkeit der Telefonate wöchentlich statt und reduzierte sich stufenweise auf ein zweiwöchiges und danach monatliches Intervall. Die Dauer der jeweils 16 durchgeführten Telefonate begann anfangs mit $60 \mathrm{~min}$ und verringerte sich auf eine 30-minutige Beratungszeit. Primäre Ergebnisse der Studie zeigten eine Belastung und teilweise depressive Symptome der pflegenden Angehörigen nach 6 Monaten auf. Die ausschließlich telefonische Beratung verbesserte die depressiven Auswirkungen und Verhaltensauffälligkeiten der Pflegenden und ist vergleichbar mit persönlichen Gesprächen [15].

In allen oben aufgeführten Studien konnte ein signifikantes Ergebnis bezüglich der Wirksamkeit der Telepflege erzielt werden. Vor einigen Jahren wurde in ersten internationalen Untersuchungen die grundsätzliche Durchführbarkeit der Tele-Health-Betreuung für Stomaträger und die im Ergebnis aus dieser Betreuungsform resultierende Versorgungsqualität im außerklinischen Bereich untersucht [16-18]. Aus pflegefachlicher Perspektive liegt es daher nahe, auch die Pflege und Versorgung von Menschen mit Fisteln und Stomaanlagen zukünftig mit digitaler Unterstützung auszuführen. Treibende Kräfte sind hier u. a. auch die Kostenträger im Gesundheitswesen. Bereits vor der Corona-Krise wurde in einem Krankenkassen-Leistungsvertrag 
die Forderung an die Leistungserbringer aufgenommen, den Stomaträgerinnen und Stomaträgern auch die Betreuung über einen Online-Zugang anzubieten. Hier wurde erstmalig die Option einer Fernbetreuung von Menschen mit einem Stoma durch Leistungserbringer im ambulanten Bereich als Vertragsbestandteil durch einen Kostenträger definiert. Unter dem starken wirtschaftlichen Druck zwischen den Leistungserbringern tritt eine notwendige Beratung von Menschen mit einem Stoma möglicherweise allerdings unter Umständen in den Hintergrund [19]. Dabei kann sich eine individuelle Beratung präventiv auf die Reduktion möglicher Stomakomplikationen mit weiteren verbundenen Versorgungskosten auswirken [20].

\section{》) In Zukunft ist eine Anpassung der Edukationsmethoden unter Nutzung digitaler Unterstützung notwendig}

In einer Fragebogenstudie 2016 untersucht die Berlin Ostomy Study die Lebensqualität und den Unterstützungsbedarf an 2647 Patientinnen und Patienten mit einem Ileo- oder Kolostoma. Dabei wurden patientenrelevante Informationen bezüglich der Stomaanlage, vorherige Behandlungen und Stomakomplikationen retrospektiv erfasst. Die Ergebnisse der Studie verweisen auf eine Verschlechterung der Lebensqualität bei Patientinnen und Patienten mit einem komplizierten Stoma. Dabei beurteilen die Befragten neben einem intensiven Kontakt ebenso Aufklärung und Betreuung am hilfreichsten [21]. Zudem erfassten die Selbsthilfegruppen der Deutschen ILCO e.V. und die Medizinische Hochschule Hannover in einer Fragebogenstudie 2005 Defizite in der Beratung von Stomaträgerinnen und Stomaträgern. Der Bezug gilt besonders bei Stomaversorgungsproblemen [22]. Aufgrund mangelnder Information und Beratung werden Stomakomplikationen häufig nicht erkannt und bleiben unbehandelt. Treten Stomakomplikationen auf, können diese neben einer Einschränkung der Lebensqualität eventuell

coloproctology 2021 · 43:223-228 https://doi.org/10.1007/s00053-021-00537-1

(c) Springer Medizin Verlag GmbH, ein Teil von Springer Nature 2021

\section{W. Droste $\cdot$ M. Wieczorek}

\section{Digitalisierungsvorschub für spezialisierte Pflege? Arbeitsbedingungen der Pflegeexperten Stoma, Kontinenz und Wunde während der SARS-CoV-2-Pandemie}

\section{Zusammenfassung}

Seit Jahren macht sich der Einfluss der fortschreitenden Digitalisierung in den Arbeitsbereichen der Pflegeexperten Stoma, Kontinenz und Wunde (PSKW) bemerkbar. Vor einigen Jahren wurde in ersten internationalen Untersuchungen die Durchführbarkeit der Tele-Health-Betreuung und die im Ergebnis aus dieser Betreuungsform resultierende Versorgungsqualität für die Stomaträgerinnen und Stomaträger im außerklinischen Bereich erprobt. Treibende Kräfte sind hier u. a. auch die Kostenträger im Gesundheitswesen, die unter Nutzung digitaler Hilfsmittel eine kostengünstige Versorgungsstruktur für die Versicherten aufbauen möchten. Bereits vor der Corona-Krise wurde in einem Krankenkassen-Leistungsvertrag die Forderung an die Leistungserbringer aufgenommen, den Stomaträgerinnen und Stomaträgern auch die Betreuung über einen Online-Zugang anzubieten. Hier wurde also erstmalig die Option einer Fernbetreuung von Menschen mit einem Stoma durch Leistungserbringer im ambulanten Bereich als Vertragsbestandteil definiert. In diesem Beitrag wird die Möglichkeit einer TelecareStomatherapie als Ergänzung zur Face-toface-Betreuung von Stomaträgerinnen und Stomaträgern durch Pflegeexperten SKW dargestellt und situativ unter der aktuellen Corona-Pandemie betrachtet.

Schlüsselwörter

Stomatherapie - Telecare - Corona-Pandemie . Versorgungsqualität · Spezialisierte Pflege

\section{Advance of digitalization for specialized care? Working conditions of the care experts in ostomy, continence and wounds during the SARS-CoV-2 pandemic}

\section{Abstract}

For years the influence of progressive digitalization has become noticeable in the work areas of care experts in ostomy, continence and wounds (PSKW). A few years ago the feasibility of tele-healthcare and the resulting quality of care for ostomates in the out of hospital area were also tested in international studies. The driving forces included the insurance companies in the healthcare system who wanted to build a cost-effective supply structure for the insured people using digital aids. Even before the corona crisis a health insurance service contract included the requirement that service providers also offer stoma care for ostomates via online access. For the first time, the option of remote care for people with an ostomy by service providers in the outpatient area was defined as part of the contract. In this article the possibility of telecare stomatherapy as a supplement to face to face care of ostomates by SKW care experts is presented and the situation under the current corona pandemic is considered.

Keywords

Stoma therapy - Telecare - Corona pandemic . Quality of care $\cdot$ Specialized care zu einer Wiedereinweisung in das Krankenhaus führen. Das bedeutet, dass ein Versorgungsmodell in optimaler Weise nicht nur bei Problemsituationen und Komplikationen, sondern insbesondere prophylaktisch eine ortsunabhängige und kontinuierliche Betreuung gewährleistet. Die notwendige Ausrichtung besteht darin, eine adäquate, kontinuierliche Beratung aller Stomaträgerinnen und Stomaträger, über den Kranken- hausaufenthalt hinaus, zu gewährleisten. Mit dem innovativen Konzept einer Telecare-Beratung könnte allen am Rehabilitationsprozess beteiligten Personen, unabhängig vom Wohnort, eine gleiche Beratungsqualität ermöglicht werden.

Während der aktuellen Corona-Pandemie sind viele strukturierte Beratungsangebote durch Pflegeexperten SKW aufgrund der veränderten Abläufe in den Gesundheitseinrichtungen auf 
der Strecke geblieben. Gleichzeitig erlebten die Pflegeexperten aber auch die nationalen und internationalen Auswirkungen der Pandemie. Fachkongresse sowie Messen und Ausstellungen wurden abgesagt, und die Kommunikation zwischen PSKW und Mitarbeiterinnen sowie Mitarbeitern aus Herstellerunternehmen und Nachsorgeunternehmen erfolgte meist nur noch aus dergebotenen Distanz. Aufgrund der Besuchsverbote in den Kliniken war auch der Besuch von Patientinnen und Patienten mit einem Stoma durch die Vertreter der Selbsthilfe nicht mehr wie gewohnt möglich. Auch die oft notwendige Anleitung pflegender Angehöriger war aufgrund der Hygienevorgaben nicht mehr realisierbar. Da es sich nicht nur um kurzfristige Auswirkungen der Pandemie handeln wird, sind zukünftig langfristige Veränderungen der bisherigen Vorgehensweisen gefragt. Hier ist eine moderne Anpassung der Edukationsmethoden unter Nutzung digitaler Unterstützung notwendig.

\section{Methoden der Telecare in der Stomatherapie}

Die Edukation von Menschen mit einem Stoma durch Stomatherapie und -versorgung ist aus heutiger Sicht nicht vollständig digitalisierbar. Der fehlende Zugang durch PSKW und auch der Stomaträgerinnen und Stomaträger zu geeigneten digitalen Anwendungen ist oft das erste Hindernis auf dem Weg der digitalen Kommunikation. Emotionale Bedenken stellen eine weitere Hürde dar. Mit dem Einsatz digitaler Technologien soll es ja nicht zu einer Entfremdung im individuellen Verhältnis zwischen PSKW und Stomaträgerinnen und Stomaträgern kommen. Telecare als neue Organisationsform soll in dieser Form keine bisher gewohnte zwischenmenschliche Pflegefachbetreuung vollständig ersetzen, sondern als optionale Ergänzung fungieren. Für PSKW ist die Verwendung von ortsunabhängigen (IKT) attraktiv.

Eine Umsetzung der Patientenedukation in Form der Teleberatung, bedarfsweise kombiniert mit einem Videokonferenzsystem, ist in der klinischen Stomatherapie in Deutschland realisierbar. Die Herausforderung besteht darin, be- sonders für Stomaträgerinnen und Stomaträger sowie allen am Rehabilitationsprozess beteiligten Akteuren ein hilfreiches, einfaches und sicheres Beratungsangebot zu realisieren. Die PSKW sind dabei mit ihrer bedeutsamen Rolle im Krankenhaus bei der detaillierten Ausarbeitung der dazu notwendigen Details aktiv gefordert. Für den Erfolg muss das zukünftige Dienstleistungsangebot allerdings von den potenziellen Nutzerinnen und Nutzern akzeptiert sein. Des Weiteren ist der Fokus auf die Gewährleistung des Datenschutzes gemäß der DSGVO bei den notwendigen IT-Systemen sowie allen Beteiligten im Prozessverlauf sicherzustellen.

Im Rahmen des Krankenhausaufenthalts könnten Patientinnen und Patienten zur Vorbereitung mit einem Teleberatungskoffer ausgestattet werden. Dieser beinhaltet eine notwendige Bedienungsanleitung sowie eine Informationsmappe mit notwendiger Einverständniserklärung zur Nutzung der erhobenen Daten gemäß Datenschutzgrundverordnung (DSGVO). Im Verlauf eines Informationsgesprächs könnten die Betroffenen in die Durchführung der Tele- und Videoberatung eingewiesen werden. Damit für die poststationäre Teleberatung genügend Zeit seitens der PSKW eingeräumt wird, ist es notwendig, hierfür vorab einen telefonischen Termin zu vereinbaren oder direkt feste Sprechzeiten einzurichten. Ebenfalls ist sicherzustellen, dass die PSKW in der Klinik auch die räumlichen Voraussetzungen vorfinden, die zur Durchführung einer Videoberatung notwendig sind. Häufig genug teilen sich heute noch im Klinikalltag mehrere Pflegekräfte einen gemeinsamen stationären Computer und nicht jede PSKW kann in aller Ruhe im eigenen Büro arbeiten.

Ein wichtiger Einsatzbereich für Telecare in der Stomatherapie könnte in der Identifikation und dem Management bei auftretenden, stomabezogenen Komplikationen bestehen. In vielen Studien wird eine allgemeine Stomakomplikationsrate von $21-70 \%$ identifiziert [23]. Für Stomaträgerinnen und Stomaträger ist eine Komplikation fast immer auch mit Einschränkungen der Lebensqualität und möglicherweise auftretenden Schmer- zen verbunden. Die Begutachtung einer auftretenden Stomakomplikation könnte exemplarisch über den Einsatz eines Videokonferenzsystems erfolgen, bei dem Betroffene zusammen mit dem Nachversorger und der PSKW im Krankenhaus kommunizieren. Bei unklarem Sachverhalt sollte selbstverständlich trotzdem eine persönliche Vorstellung bei der PSKW erfolgen.

\section{Ausblick}

Telecare umfasst Einsatz- und Anwendungsarten pflegefachlicher Versorgungskonzepte bzw. Dienstleistungen mit einem direkten Patientenbezug [4]. Im Rahmen einer raumunabhängigen Konsultation über Telefon und Video könnten PSKW auf dem Fachgebiet der Stomatherapie ihre Expertise in beratender Form zur Verfügung stellen. Mit einer Teleberatung in der klinischen Stomatherapie könnten Stomaträgerinnen und Stomaträger sowie deren Angehörige in der häuslichen Umgebung weiterhin persönlich, aber auf Distanz betreut werden. Dazu soll bei der Ausgestaltung von Telecare besonders für alle am Rehabilitationsprozess beteiligten Akteure ein hilfreiches, einfaches und sicheres Beratungsangebot realisiert werden. In allen bisherigen Studien ist eine signifikante Verbesserung in der Beratung, Gesundheitsversorgung sowie der Patientenzufriedenheit nachzuweisen. In diesem Zusammenhang zeigt der Einsatz von modernen IKT im Gesundheitswesen einen positiven Effekt auf Patientinnen und Patienten wie auch Angehörige. Die Auswirkungen sind in der Kosteneffektivität und niedrigeren Krankenhausaufenthaltsraten mit nachfolgender Kostenersparnis klar zu erkennen: Aufgrund der bisher mangelnden Anwendung des innovativen Konzeptes in der Pflege, mit Fokus auf Stomaträgerinnen und Stomaträger, mangelt es bisher an aussagekräftigen Untersuchungsergebnissen. Die Anwendung von Teleberatung in der Stomatherapie stellt eine denkbare und sinnvolle Ergänzung der Kommunikation zwischen dem häuslichen Bereich und dem Krankenhaus dar. Somit kann die Teleberatung dazu beitragen, die 
Pflege auch in Zukunft auf einem bedarfsgerechten Niveau zu gewährleisten. Selbstverständlich soll die zukünftige Stomatherapie nicht ausschließlich in Form von Telecare stattfinden. Schon mit Blick auf die Empfangsbereitschaft und Teilnahmemöglichkeit vieler Menschen mit einer Stomaanlage an TelecareStomatherapie-Vorhaben sind der Umsetzbarkeit hier Grenzen gesetzt. Vielmehr ist hier eine ideale Verknüpfung von persönlicher Beratung in der Präsenz vor Ort mit nachfolgender Unterstützung in der Telecare-Stomatherapie zu sehen. Ergänzung statt Ersatz. Für die Pflegeforschung bietet sich dadurch eine Chance, neue Projekte im Rahmen der Teleberatung durchzuführen, um der Angst vor Veränderung wissenschaftlich begründet zu begegnen.

\section{Fazit für die Praxis}

- Durch Telecare könnten Stomaträgerinnen und Stomaträger im Rahmen der Stomatherapie in ihrer häuslichen Umgebung weiterhin persönlich, aber auf Distanz betreut werden.

- Diese Betreuungsform spielt insbesondere während der COVID-19Pandemie eine zunehmend bedeutende Rolle.

- Die Telefonberatung soll nicht den persönlichen Kontakt ersetzen, stellt aber als Ergänzung der Stomatherapie ein sinnvolles und hilfreiches Instrument für die Pflegefachkräfte SKW dar und kann zur Verbesserung der Versorgungsqualität beitragen.

- Der Einsatz von e-Health wird allerdings durch die eingeschränkte Teilnahmefähigkeit der Patienten limitiert, die keinen Zugang zu digitalen Kommunikationsmitteln haben.
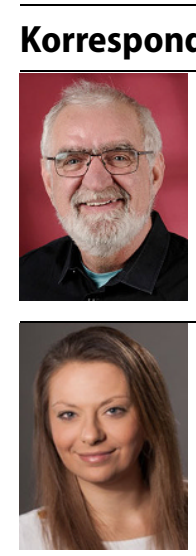

\section{Margarete Wieczorek} FgSKW e.V.

Nikolaus-Gross-Weg 6, 59379 Selm, Deutschland pskw@margaretewieczorek.de

Werner Droste Seminare \& Beratung, Pflegeexperte SKW, Selm, Deutschland.

Margarete Wieczorek Akademische Kontinenz- und Stomaberaterin, Pflegeexpertin SKW, Regensburg, Deutschland.

\section{Einhaltung ethischer Richtlinien}

Interessenkonflikt. W. Droste und M. Wieczorek sind jeweils als selbstständige und freiberufliche Berater und Dozenten tätig. Beide erhalten für ihre Tätigkeiten regelmäßig Honorare von Herstellerunternehmen, Homecare-Unternehmen sowie von Bildungseinrichtungen. W. Droste und M. Wieczorek geben an, dass kein Interessenkonflikt besteht.

Für diesen Beitrag wurden von den Autoren keine Studien an Menschen oder Tieren durchgeführt. Für die aufgeführten Studien gelten die jeweils dort angegebenen ethischen Richtlinien.

\section{Literatur}

1. Fallpauschalenbezogene Krankenhausstatistik (DRG-Statistik) für 2019; @ Statistisches Bundesamt (Destatis), 2020

2. Berufsbild für Pflegeexperten Stoma, Kontinenz und Wunde. https://www.fgskw.org/berufsbild. php;.Zugegriffen: 14. Nov. 2020

3. AGnES - Hausarztunterstützung durch qualifizierte Praxismitarbeiter - Evaluation der Modellprojekte: Qualität und Akzeptanz; Dtsch Arztebl Int 2009; 106(1-2): 3-9; https://doi.org/10.3238/ arztebl.2009.0003; Berg, Neeltje van den; Meinke Claudia; Heymann, Romy; Fiß, Thomas; Suckert, Eileen; Pöller, Christian; Dreier, Adina; Rogalski, Hagen; Karopka, Thomas; Oppermann, Roman; Hoffmann, Wolfgang

4. Fischer F, Aust V, Krämer A (2016) eHealth in Deutschland, Anforderung und Potenziale innovativer Versorgungsstrukturen. Heidelberg, Bd.4. Springer, Berlin

5. Bundesärztekammer. https://www.bundesaerzte kammer.de/aerzte/telematiktelemedizin/tele medizin/ (Stand: 7. Aug. 2018). Zugegriffen: 12. Okt. 2020

6. Dietzel GTW (2004) Auf dem Weg zur europäischen Gesundheitskarte und zum e-Rezept. In: Jähn K, Nagel E (Hrsg) e-Health. Springer, Berlin:, S2-6
7. Jähn Karl; Reiher Michael, Braasch Paul; Ebert Michael (2007): E-Health. In: Nagel Eckhard (Hrsg): Das Gesundheitswesen in Deutschland. Struktur, Leistungen, Weiterentwicklungen. 4. Auflage, Deutscher Ärtze-Verlag, Köln.

8. Niederberger-Burgherr J (2007) (2007): Telenursing/Telepflege. Über das Videotelefon in direkter Verbindung. In. Krankenpflege, Bd. 4, S 10-14

9. Reese Robert; Conoley Collie; Brossart Daniel (2006):Theattractiveness of telephone counseling an empirical investigation of client perceptions. Journal of Counseling \& Development; 84:54-60.

10. Dochterman, Joanne; Bulechek, Gloria; Butcher, Howard; Wagner Cheryl (2004): Nursing Interventions Classification (NIC) 4th edition Intervention Labels \& Definitions. St. Louis, MO: Mosby: http://www.seapaonline.org/ uploads/documentacion/Metodolog\%C3\%ADa \%20Enfermera/labeldefinitions-NIC.pdf (Stand: 31. Aug. 2018). Zugegriffen: $12.0 \mathrm{kt} .2020$

11. Riegel B, Carlson B, Kopp Z (2002) Effect of Standardized Nurse Case-Management Telephone Intervention on Resource Use in Patients with Chronic Heart Failure. Arch Intern Med 25:705-712

12. O'Reilly R, Bishop J, Maddox K, Hutchinson L, Fisman M, Takhar J (2007) Is telepsychiatry equivalent to face-to-face psychiatry? Results from a randomized controlled equivalence trial. Psychiatr Serv 58(6):836-843

13. Hartford K (2005) Telenursing and patients' recovery from bypass surgery. J Adv Nurs 50(5):459-468

14. Piette J, Weinberger M, McPhee S (2000) The effect of automated calls with telephone nurse followup on patient-centered outcomes of diabetes care: a randomized, controlled trial. Med Care 38:218-230

15. Tremont Geoffrey; Davis Jennifer; Papandonatos George; Ott Brian; Fortinsky Richard; Gozalo Pedro; Yue Mun; Bryant Kimberly; Grover Christine; Bishop Duane. Psychosocial Telephone Intervention for Dementia Caregivers: A Randomized, Controlled Trial: 541-548. Online Im WWW unter URL: https://www.ncbi.nlm.nih.gov/pmc/articles/ PMC4306648/.Zugegriffen: 12. Okt. 2020

16. Zhang, J. E., Wong, F. K. Y., You, L. M., \& Zheng M. C. (2012). A qualitative study exploring the nurse telephone follow-up of patients returning home with a colostomy. Journal of Clinical Nursing, 21(9-10), 1407-1415. https://doi.org/10.1111/j. 1365-2702.2011.03824.x

17. SK, B., McDonald, P., AM, L., Krupinski, E., \& Blackett, A. (2004). Traditional versus telenursing outpatient management of patients with cancer with new ostomies. Oncology Nursing Forum, 31(5), 1005-10106p. https://doi.org/10.1188/04. ONF.1005-1010

18. Sun, V., Ercolano, E., McCorkle, R., Grant, M., Wendel, C. S., Tallman, N. J., Passero, F., Raza, S., Cidav, Z., Holcomb, M., Weinstein, R. S., Hornbrook, M. C., \& Krouse, R. S. (2018). Ostomy telehealth for cancer survivors: Design of the Ostomy Self-management Training (OSMT) randomized trial. Contemporary Clinical Trials, 64(July 2017), 167-172. https://doi. org/10.1016/j.cct.2017.10.008

19. Deutsche ILCO e.V. (Hg.)(2016): Hilfsmittelversorgung. Gesetze seit 2007 infolge von Gesundheitsreformen mit Auswirkungen auf die Stomaversorgung. Online im WWW unter: https:// www.ilco.de/fileadmin/user_upload/SozInfo_ Hilfsmittelversorgung_2016.pdf.Zugegriffen: 13. Okt. 2020

20. Sauer, Kristin.; Kemper Claudia.; Schulze Jana; Glaeske Gerd (2013): Barmer GEK Heil- und 
Hilfsmittelreport 2013. Auswertungsergebnisse der BARMER GEK Heil- und Hilfsmitteldaten aus den Jahren 2011 bis 2012. Hg. Barmer GEK. Asgard Verlagsservice $\mathrm{GmbH}$, Siegburg. Online im WWW unter: https://www.barmer.de/blob/ 37426/1b7a43d86cf4791d9f778bbb9d971f58/ data/pdf-heil-und-hilfsmittelreport-2013.pdf. Zugegriffen: 10. Okt. 2015

21. Braumann, C.; Müller, V.; Knies, M.; Aufmesser, B.; Schwenk, W.; Koplin G. (2016): Quality of life and need for care in patients with an ostomy: a survey of 2647 patients of the Berlin OStomyStudy (BOSS). Langenbeck's Archives of Surgery Volume 401, Issue 8: 1191-1201. Online im WWW unter: https://link.springer.com/article/10.1007 \%2Fs00423-016-1507-z

22. Deutsche ILCO e.V.; Medizinischen Hochschule Hannover (2004): Die ambulante Versorgung von Stomaträgerinnen und -trägern. Ein Kooperationsprojekt der Deutschen ILCO e.V. und der Medizinischen Hochschule Hannover (MHH). Arbeitsbereich Medizinsoziologie. Online im WWW unter: https://slidex.tips/download/dieambulante-versorgung-von-stomatrgerinnenund-trgern.Zugegriffen: 15. Dez. 2017

23. Shabbir J, Britton DC (2010) Stoma complications: a literature overview. Colorectal Dis 12:958-964

\section{Fachnachrichten}

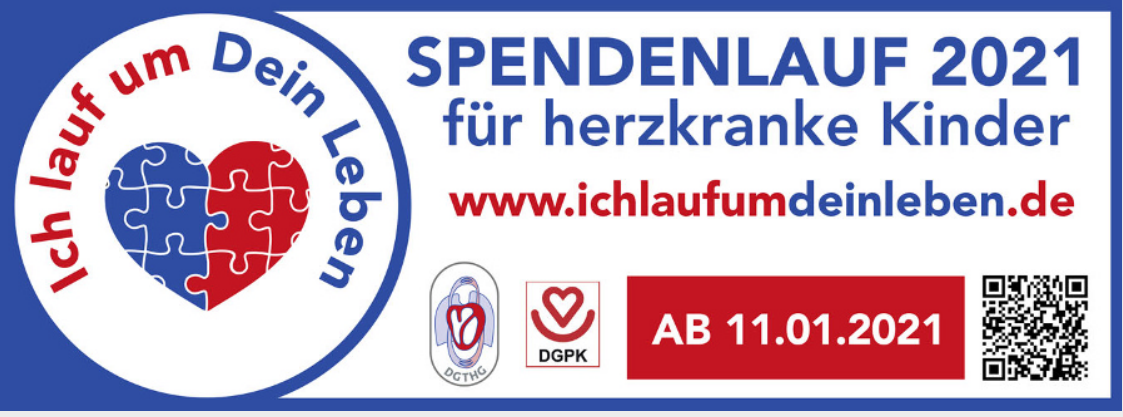

\section{Ich lauf um Dein Leben - Spendenlauf für Kinderherz- transplantationen}

Mit der Aktion „Ich lauf um Dein Leben" rufen die Deutsche Gesellschaft für Thorax-, Herz- und Gefäßchirurgie e. V. (DGTHG) und die Deutsche Gesellschaft für Pädiatrische Kardiologie und Angeborene Herzfehler e. V. (DGPK) zu einem virtuellen Spendenlauf auf. Mit dem Spendenlauf sollen Kinder unterstützt werden, die dringend eine Herztransplantation brauchen.

Schwerst herzkranke Kinder warten häufig viele Monate bzw. mehr als ein Jahr auf ein passendes Spenderherz. Aktuell sind es 50 Mädchen und Jungen bundesweit. Andererseits werden weniger als 30 Spenderherzen jährlich in Deutschland transplantiert. Die Herausforderungen sowie Ängste und Sorgen, mit denen die kleinen Patientinnen und Patienten mit ihren Familien leben müssen, sind immens.

Um auf dieses wichtige Thema besonders aufmerksam zu machen, haben die Deutsche Gesellschaft für Thorax-, Herzund Gefäßchirurgie e.V. (DGTHG) und die Deutsche Gesellschaft für Pädiatrische Kardiologie und Angeborene Herzfehler e.V. (DGPK) daher am 11. Januar 2021 einen virtuellen Spendenlauf gestartet. Damit wollen die herzmedizinischen Fachgesellschaften im Rahmen ihrer digitalen Jahrestagungen Herzmedizin 2021 speziell das Thema Kinderherztransplantationen ins Bewusstsein der Öffentlichkeit rufen, um durch die Spenden Kindern, die ein Spenderorgan benötigen, noch gezielter helfen zu können. Der Erlös wird das bundesweit tätige Aktionsbündnis Angeborene Herzfehler e.V. unterstützen, in dem sechs Patientenvertretungen für angeborene Herzfehler vereint sind, um mit gemeinsamen Kräften Kindern, Jugendlichen und Erwachsenen mit angeborenen Herzfehlern zu helfen.

Mitmachen kann jeder: Der Erwerb des "Ich-lauf-um-dein-LebenLaufshirts" (https://www.tshirt-drucker.de/c/spendenlauf) impliziert bereits eine Spende und stattet die Läufer*innen passend für diesen Anlass aus; ebenfalls möglich und willkommen ist auch eine direkte Spende.

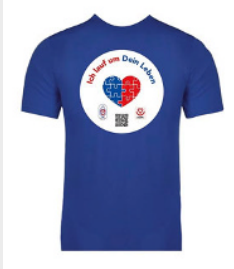

Der finale Jahreserlös der Charity-Aktion wird zum 31.12.2021 vorgestellt.

Alle Informationen erhalten Sie unter: https://www.ichlaufumdeinleben.de

\section{Spendenkonto Kinderherztrans- plantationen (DGTHG):}

Kontoinhaber:

Deutsche Gesellschaft für Thorax-, Herz- und Gefäßchirurgie e.V. (DGTHG)

Pax-Bank eG

IBAN: DE64 370601936005727004

BIC: GENODED1PAX

Stichwort: „Ich lauf um Dein Leben 2021"

\section{Weitere Informationen unter:} https://www.dgthg-jahrestagung.de http://www.kinderkardiologie.org/ jahrestagung/ 